\title{
3D MODEL-BASED TIME GATHER VELOCITY ANALYSIS
}

STUART W. FAGIN', ALEX LITVIN' and AL BROWN ${ }^{2}$

' Paradigm Geophysical Corp., Houston TX 77060, USA

2 Texaco Corp.

Highly accurate earth models are required before one can experience the benefits of depth imaging. As structure and prospect become increasingly complex and subtle, Dix-based methods become ineffective as velocity estimation tools. Model-based methods are more effective because they bring the interpreter's evolving understanding of structure to the velocity estimation process. We describe the use of two types of model-based methods employed in three dimensions; stacking value matching and coherency inversion. These methods are used to define interval velocities on a land 3D survey.

In stacking value matching predicted moveout is computed from a model for a range of velocities and the velocity which best matches real moveout, as described by the stacking value, is identified. In this method we recognize that stacking values are not direct measures of intrinsic subsurface velocities, but are regarded simply as labels for hyperbolas which best describe moveout. The application of this method is well established in two dimensions (Gerritsma, 1977). In three dimensions we have the added consideration of source-receiver azimuth. Maps are made of stacking velocity for individual azimuth, which should match the dominant azimuth of the real gather. In coherency inversion, predicted moveout is computed from a model for a range of velocities, and the velocity which best describes coherent reflections within the gather is identified. In three dimensions care must be taken to properly emulate the variable sourcereceiver azimuth within the gather, particularly in land data sets. The relative advantages of each method are contrasted and tested. 
